\title{
Pulse Thermography for Detection of Differentiated Tissue
}

by M. Strojnik and G. Paez

Centro de Investigaciones en Óptica, A.P. 1-948, C.P. 37000, León, Guanajuato, México, mstrojnikp@aol.com

\section{Abstract}

We report on the study of the relationship between the length of the pulses and the depth of the boundary to be detected in thermally transmissive tissues within the therapeutic window, red to IR spectral range. We use a three-layer theory to predict the possibility of detection of an abnormal growth.

\section{Shallow cancerous growth (in males)}

One of the first applications of the thermography in the seventies was the diagnosis of the breast center. In the following thirty years, novel and more sensitive thermographic cameras became available, often used for concurrent and complementary diagnosis with the $x$-ray imaging.[1, 2] The survival rate of this type of cancer has shown significant improvement in women, while it has an appreciably lower 5-year survival rate in men than in women, even though the lesion location is shallower for men.

\section{Methodology: experimental concepts}

We evaluate the features of pulse thermography to identify possible lesions under the skin, in-vivo. We model a tissue with a tumor-like growth as a three-layer object, with the thickness of the third layer appreciably larger than that of the first two. When the defect is absent the second layer merges into the neighboring ones. We set up the experimental setup as illustrated in Figure 1.

X-ray transmissive and reflective imaging, ultrasound, and MRI are traditional radiation-based techniques to find a tumor. Likewise, the IR pulse thermography $[3,4]$ relies on the transmission of IR radiation through the tissue, until it encounters a boundary with different physical and optical characteristics. There, it is reflected and it travels back to the front surface. It is seen as a thermal wave at the surface of the object under test. This concept is illustrated schematically in Figure 2.[5] The obvious advantage of the detection of abnormal tissue in-vivo with IR radiation is its human-friendliness. Additionally, the living tissue is may be probed within the so-called therapeutic spectral interval, encompassing red to near IR [6], permitting transmission of illuminating radiation up to the point where a boundary between two optically/ physically different materials is encountered.

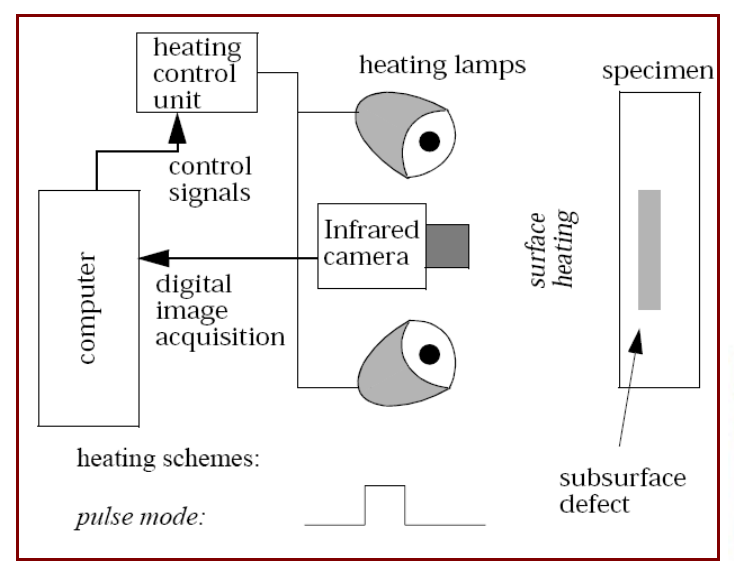

Fig. 1. Principal components of our experimental setup to perform the pulse thermography.[5]
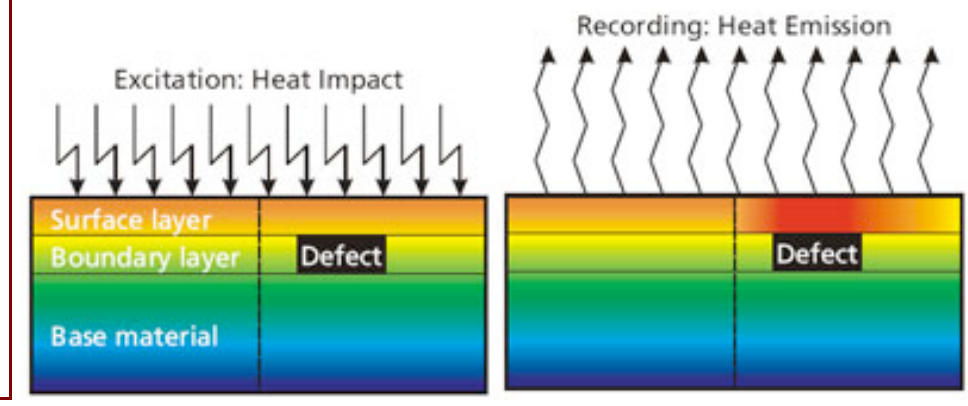

Fig. 2. We can model a tissue with a tumor-like growth as a threelayer object, with the thickness of the third layer very large.

\section{Methodology: theoretical concepts}

When the surface of the object under study is illuminated with visible/ near IR collimated series of light pulses, the surface transmits the radiation that travels through the medium. At an interface between two physically different media, the thermal pulse is reflected, travelling back to the front surface. The IR camera recording sequence of frames 
detects it a thermal wave. Figure 3 illustrates this concept for two pixels, in the case of infinitely thick object under study, allowing a 1-D heat-transfer treatment.[7] The illuminated surface undergoes a significant temperature increase only when the thermal wave is reflected from some buried inhomogeneity, indicating a change in physical/ thermal characteristics. When there is no buried boundary between layers, the pixel surface temperature is slightly increased and constant $(\Delta \theta=0)$. When there exists a defect, the pixel surface temperature increases rapidly during the pulse duration, achieving its maximum value at the end of the pulse. Thus, optimum pulse duration must be adjusted to the anticipated depth of the defect. Figure 3 [5] illustrates normalized pixel temperature increase vs normalized observation of the temperature increase. This diagram may also be interpreted as showing two representative pixels in successive series of the frames: one encountering buried obstruction, and one freely propagating through the infinitely thick object.

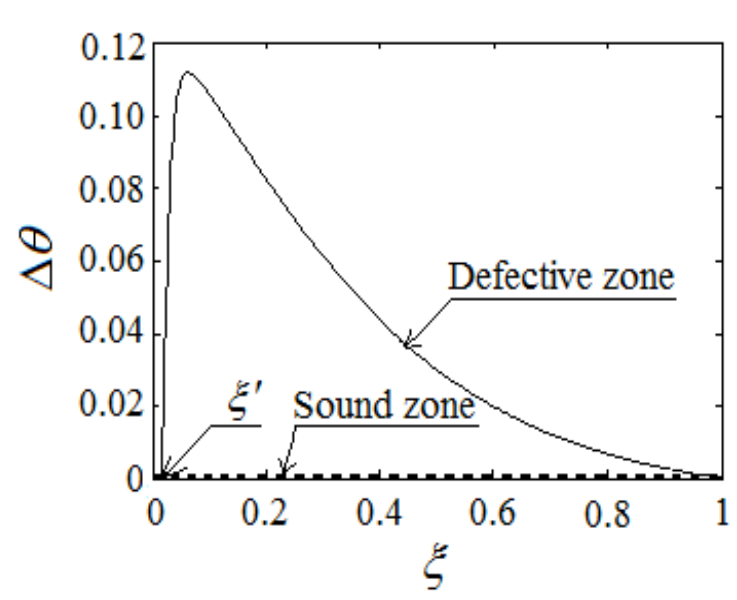

Fig. 3. Normalized pixel temperature increase vs normalized observation time of the temperature increase (proportional to frame number).

$$
\Delta \theta(x, y, \xi)=\left|\theta(x, y, \xi)-\theta_{R}(\xi)\right|
$$

Here $\theta(x, y)$ is normalized reference temperature increase at surface point $P(x, y)$. It may be found for the layered material as a sum of exponentials.

$$
\theta(\xi)=1+\sum_{i=1}^{j} C_{i} e^{-\xi / \psi_{i}}
$$

Here we introduce constants.

$$
\begin{aligned}
& \psi_{i}=\frac{1}{-\alpha_{i} B_{i}^{2} t_{f}} \quad a=\frac{T_{0}}{T_{0}-T_{f}} \\
& \xi(t)=t / t_{f}
\end{aligned}
$$

$\xi$ is normalized time. $t_{f}$ is the time constant; $\xi(x, y)^{\prime}$ is the normalized time for the irradiation pulse to travel to the defect at Pixel $\mathrm{P}(\mathrm{x}, \mathrm{y})$; $\xi(\mathrm{x}, \mathrm{y})$ is normalized time for the irradiation pulse to travel from the defect to the front surface.

The defect depth is actually not of primary significance in these studies - we are trying to ascertain just the existence of the defect. However, the depth at which the reflection occurs is easily found for pixel $P(x, y)$ as a function ofsurface coordinates. This is particularly so for a relatively flat surface, such as a chest of a male.

$$
d(x, y) \approx \sqrt{\alpha t_{f} \xi^{\prime}(x, y)}
$$

Here $\alpha_{i}$ is the thermal diffusivity in [ $\mathrm{m}^{2} / \mathrm{s}$ ] of a layer, defined as $\alpha_{i}=k_{i} /\left(\rho_{i} c_{p i}\right)$, where $\mathrm{k}_{\mathrm{i}}, \rho_{\mathrm{i}}$, and $\mathrm{p}_{\mathrm{i}}$ are the thermal heat conductivity, density, and heat capacity of the $\mathrm{i}^{\text {th }}$ layer.

\section{Summary}

We report on the study of the relationship between the length of the pulses and the depth of the boundary to be detected in thermally transmissive tissues within the therapeutic window, red to near IR spectral range. We use a threelayer theory to assess the possibility of detection of an abnormal tissue growth. We comment on the applicability of the method to detection of buried boundaries with different radii of curvature.

\section{REFERENCES}

[1] Belliveau N., Keyserlingk J., "Infrared Imaging of the Breast: Initial Reappraisal Using High-Resolution Digital Technology in 100 Successive Cases of Stage I and II Breast Cancer," Breast Journal, 1998; 4 (4).

[2] http://www.cancer.org/Cancer/BreastCancer/DetailedGuide/breast-cancer-survival-by-stage

[3] Scholl M. S., "Target Temperature Distribution Generated and Maintained by a Scanning Laser Beam," Appl. Opt. Vol. 21 (12), 2146-2152 (1982).

[4] Cielo P., "Pulsed photothermal evaluation of layered materials," J. Appl. Phys. Vol. 56, $230-234$ (1984).

[5] Ramirez-Granados J. C., Paez G., and Strojnik M., "Reconstruction and analysis of pulsed thermographic sequences for nondestructive testing of layered materials," Appl. Opt. Vol 49 (9), 1494-1502 (2010).

[6] Vasquez-Jaccaud C., Paez G., Strojnik M., "Wavelength selection method with standard deviation: application to pulse oximetry," Annals of Biomedical Engineering Vol 39 (7), pp 1994-2009 (2011).

[7] Scholl M. S., "Spatial and Temporal Effects due to Target Irradiation: A Study," Appl. Opt. Vol 21 (9), 1615-1620 (1982). 